\title{
Cardiac catheterization and complications: initial experience
}

\author{
L. Dubey ${ }^{1}$, S. K. Sharma ${ }^{1}$ \\ DM Resident,Cardiology, College of Medical Sciences and Teaching Hospital, Bharatpur, Chitwan, Nepal
}

\begin{abstract}
Cardiac catheterization for diagnostic and therapeutic purposes has been routinely used since last one year in College of Medical Sciences and Teaching Hospital, Bharatpur, Nepal. Because all cardiac catheterizations involve the insertion of cardiac catheters into the circulatory system, it should not be surprising that a variety of complications can ensue. These complications range from minor problems with no long-term sequelae to major problem even death.
\end{abstract}

A total of 357 consecutive diagnostic and therapeutic cardiac catheterization performed in College of Medical Sciences and Teaching Hospital, Bharatpur between April 2011 to April 2012 were evaluated for their complications. Among them 220 (61.6\%) were coronary angiogram, 65 (18.2\%) percutaneous transluminal coronary angioplasty (PTCA) and stenting, 7 ( $1.9 \%)$ permanent pacemaker insertion, 65 $(18.2 \%)$ were others including temporary pacemaker insertion, peripheral angiography and carotid angiography.

There were 3 deaths $(0.84 \%)$. Two deaths occurred following coronary angiogram and 1 death following PTCA stenting. Vascular complications occurred in 5 (1.4\% patients) with groin haematoma in all. Contrast allergy occurred in $9(2.5 \%)$, vasovagal reaction in $2(0.56 \%)$, pyrogen reaction in $6(1.6 \%)$, and contrast induced nephropathy occurred in $3(0.84 \%)$ patients.

Cardiac catheterization procedure in CMS-TH, Bharatpur has acceptable low complications including death.

\section{Key words: Cardiac catheterization, complications, CMS-TH.}

\section{Introduction}

Cardiac catheterizationis an invasive intervention that is being increasingly used both for diagnosis and treatment. In 1929, Werner Forssmann was the first to advance a catheter into the heart. These days, cardiac catheterization is performed routinely in

Correspondence: Laxman Dubey

E-mail:dubeylax@yahoo.com hospitals all around the world. Catheterization laboratory was established in College of Medical Sciences, Teaching Hospital (CMS-TH), Bharatpur, Nepal in the early 2011. Since then we are routinely performing both diagnostic and therapeutic catheterizations. With improvement in technology and experience, pharmacologic development, the indications for this procedure are increasing. The 
decision to perform cardiac catheterization must be based on a careful balance of risk of procedure against the anticipated benefit to the patient. These invasive procedures hence are associated with complications.

Although diagnostic catheterizations are done by skin puncture under local anesthesia, all such procedures, both diagnostic and therapeutic, are associated with some risk. Cardiac catheterizations may lead to several minor complications such as temporary pain, nausea/ vomiting, bleeding, hematoma, contrast allergy as well as major complications such as myocardial infarction, major embolic events and death. ${ }^{1}$ Complication rate may be significantly lower in recent years because advanced catheter technologies have improved cardiac catheterization significantly.

We aim to study the catheterization procedures and their complications and hence its safety and anticipated risk in our context.

\section{Materials and methods}

We analyzed 357 consecutive patients, ranging from 19 years to 92 years old (mean, $45 \pm 10$ years), who underwent diagnostic as well as therapeutic cardiac catheterization between April 2011 to April 2012 in CMS-TH, Bharatpur. Cardiac catheterization was done both from femoral and radial arteries access site. Diagnostic and therapeutic cardiac catheterization procedures include coronary angiography, peripheral angiography, carotid angiography, temporary pacemaker insertion, percutaneous transluminal coronary angioplasty (PTCA) with stenting (including primary PTCA), and permanent pacemaker insertion. (Table 1)

Ninety two percentages of procedures were done through femoral and $8 \%$ cases were done through radial arterial access. Following procedure the femoral arterial access site was compressed manually for at least 20 minutes and the patient was admitted in wards keeping the access site leg immobilized for $6 \mathrm{hrs}$. In case of access through radial artery, radial sheath was removed immediately and the access site was compressed using bandage. The vascular complications associated with the procedures were evaluated.

Both written and informed consents were taken before the procedure. All periprocedural complications during catheterization and hospital stay were recorded.

\section{Table 1: Cardiac catheterization procedures}

\begin{tabular}{lll}
\hline S. No. & Procedure & Total \\
\hline 1. & Coronary angiography & 220 \\
2. & PTCA stenting & 65 \\
3. & Permanent pacemaker insertion & 7 \\
4. & Temporary pacemaker insertion & 18 \\
5. & Peripheral angiography & 47 \\
\hline 6. & TOTAL & 357 \\
\hline
\end{tabular}


L. Dubey et al. Cardiac catheterization and complications: initial experience

\section{Results}

Complication occurred in the procedure are given in

Table 2. The major complication of diagnostic catheterization was death and occurred in 3 patients $(0.84 \%)$ of total 357 procedures. Two deaths occurred following coronary angiogram and 1 patient died following PTCA stenting to left circumflex artery who developed retroperitoneal hematoma.

\section{Table 2: Complications}

\begin{tabular}{ll}
\hline Death & 3 \\
Vascular complications & 5 \\
Contrast allergy & 9 \\
Vasovagal reaction & 2 \\
Pyrogen reaction & 6 \\
Contrast induced nephropathy & 3 \\
\hline
\end{tabular}

Contrast allergy was the commonest complication occurred in 9 patients $(2.5 \%)$. All these patients were managed medically with antihistamines.

Several studies have reported that reaction to the iodinated contrast agent occurs in up to one percent of patients. ${ }^{2,3}$ The risk of such a reaction is increased in patients who have other atopic disorders, allergy to seafood (which contains organic iodine), or a history of prior contrast reactions. Our patients develop mild form of contrast allergies which were managed medically with antihistamines only.

Vascular access site complications (such as groin haematoma) occurred in 5 patients $(1.4 \%)$. Of those vascular complications 1 patient developed retroperitoneal hematoma next day after a successful PTCA stenting.

Three patients $(0.84 \%)$ died after the procedure. One patient (84-years of age) who underwent PTCA stenting to left circumflex artery died next day due to massive retroperitoneal hematoma. The rest 2 patients who died had acute myocardial infarction complicated with cardiogenic shock, in which coronary angiogram showed diffuse multivessel disease who were not suitable candidates for revascularization.

Antibiotics are not needed routinely when cardiac catheterization is performed with usual sterile precautions, we still give Inj. cephazolin ( $1 \mathrm{~g}$ during procedure and every 8 hours for 24 hours ) whenever we perform a delayed intervention by exchanging sheaths that were placed in an earlier diagnostic procedure or when any break in sterile technique is suspected. Despite that, pyrogen reaction manifested by fever and chill occurred in 6 patients $(1.6 \%)$. Vasovagal reaction induced by pain occurred in 2 patients $(0.56 \%)$.

\section{Discussion}

During the 1960s and 1970s, cardiac catheterization was primarily a diagnostic procedure used to evaluate hemodynamics, ventricular function and coronary anatomy. In the 1980s, the introduction of improved angioplasty equipment and new interventional devices made catheterization a much more important tool in the treatment of cardiovascular disease. Cardiac catheterization, whether diagnostic or therapeutic, invariably include both a chance of benefit and a chance of harm to the patient. The acceptable ratio of one to the other will vary, depending on the value of the procedure and the seriousness of the disease. ${ }^{4}$

Death following diagnostic catheterization is a major complication. Death as a complication of cardiac catheterization has declined progressively over the last 30 years. ${ }^{5}$ A number of baseline variables (including 
NYHA class, multivessel disease, congestive heart failure, and renal insufficiency) were identified, whose presence predicted an up to eight-fold increase in major complication rates (from 0.3 in patients with none of these factors to $2.5 \%) .{ }^{6}$ Our study showed 3 deaths $(0.84 \%)$ following 357 cardiac catheterization procedures. The mortality associated with coronary angiography has improved considerably. Previous rate was in excess of $1 \%$ in many laboratories, but with widespread use of heparinization the death rate with coronary angiography has fallen to its current low level of 0.1 to $0.3 \%,{ }^{7}$ depending on case mix. Among 3 deaths, 1 patient died because of massive retroperitoneal bleeding. The patient was an 84 -year old female who developed retroperitoneal hematoma after PTCA stenting to left circumflex artery. That patient died despite massive blood transfusion. The rest 2 patients who died had acute myocardial infarction complicated with cardiogenic shock, in which coronary angiogram showed diffuse multivessel disease who were not suitable candidates for revascularization. In a study the mortality rate for diagnostic catheterization was $0.12 \%$ in 1,609 diagnostic catheterizations. ${ }^{1}$

Vascular complication at access site was also common and occurred in 5 patients (1.4\%). Femoral artery thrombosis requires urgent surgical intervention. Pseudo aneurysm is painful and may rupture. Improvements in technique and technology have reduced the morbidity of routine diagnostic coronary angiography, but occasional mortality seems to be unavoidable. ${ }^{8}$ Many series report a mortality of between $0.1 \%$ and $0.2 \% .^{3,9,10}$ In a total of 10,271 consecutive patients undergoing cardiac catheterization who were followed up prospectively over a period of three years, the incidence of iatrogenic arteriovenous fistula was found to be $0.86 \%$.The independent risk factors for arteriovenous fistula were identified as high heparindosage, coumadin therapy, puncture of the left groin ,arterial hypertension and female gender. ${ }^{10}$ However, none of our patients develop pseudoaneurysm or arteriovenous fistula.

Several studies have reported that reaction to the iodinated contrast agent occurs in up to one percent of patients ${ }^{2,3}$ The risk of such a reaction is increased in patients who have other atopic disorders, allergy to seafood (which contains organic iodine), or a history of prior contrast reactions. Contrast allergy occurred in 9 patients $(2.5 \%)$. This was of mild form in all 9 patients. The incidence of contrast media complications in the catheterization laboratory is $0.23 \%$ with 1 death per 55,000. ${ }^{1}$ Anaphylactoid reactions are non-immune mediated, but histamine release and other mediators produce a clinical presentation indistinguishable from anaphylaxis. ${ }^{11}$

Vasovagal reaction developed in $2(0.56 \%)$ patients. Vasovagal reaction is common and may be serious if prolonged hypotension and bradycardia are unnoticed and untreated; this was precipitated by pain in a very anxious patient but responded to intravenous injection of atropine and normal saline. Pyrogen reaction manifestated by fever and rigor were also noted in 6 (1.6\%) patients. Due to cost factor, instruments like coronary guidewires, vascular sheaths, catheter etc. are reused after proper sterilization in developing country like ours. The source could be foreign protein, endotoxin or other antigenically active substance into blood. 
L. Dubey et al. Cardiac catheterization and complications: initial experience

Contrast induced nephropathy developed in $3(0.84 \%)$ patients. At least five percent of patients who undergo cardiac catheterization experience a transient rise in the plasma creatinine concentration of more than 1.0 $\mathrm{mg} / \mathrm{dL}(88 \mu \mathrm{mol} / \mathrm{L})$ due to contrast-induced renal dysfunction. ${ }^{12}$ Factors predisposing patients to this complication include diabetes mellitus, preexisting renal dysfunction, volume depletion (including low perfusion due to congestive heart failure), multiple myeloma, or the presence of other nephrotoxic medications..$^{13}$ The plasma creatinine concentration usually returns to baseline within seven days, and fewer than one percent of patients, usually those with underlying severe renal failure, go on to require chronic hemodialysis. ${ }^{12,13}$ Among those who developed contrast induced nephropathy, 2 patients recovered on conservative management, however, one patient required hemodialysis. After 3 sessions of hemodialysis, she recovered her renal function to normal.

\section{Study limitations.}

Attempts have been made to include all reported complications including death. However, minor transient complications like transient arrhythmias, hypotension may all not be recorded and missed. Finally, we did not evaluate vascular complications after discharge from the hospital.

\section{Conclusions}

Both diagnostic \& therapeutic cardiac catheterization procedures in CMS-TH, Bharatpur has acceptable low complications even in its initial phase. Low complications not only ensure patient safety and comfort, but also reduce costs and improve efficiency of cardiac catheterization.

\section{References}

1. R.M. Wyman, R.D. Safian, V. Portway, et al. Current complications of diagnostic and therapeutic cardiac catheterization. J Am Coll Cardiol. 1988;12:1400-6.

2. G. Dorros, M.J. Cowley, J. Simpson, et al. Percutaneous transluminal coronary angioplasty: Report of complications from the National Heart, Lung, and Blood Institute PTCA registry. Circulation.1983;67:723-30.

3. J.W. Kennedy. Complications associated with cardiac catheterization and angiography. Cathet Cardiovasc Diagn 1982; 8:5-11.

4. B.C. Morton, D.S. Beanlands. Complications of cardiac catheterization:one centre's experience. CMAJ 1984;131:889-92.

5. D.S. Baim. Grossman's Cardiac catheterization, angiography, and intervention. Seventh Edn 2006;36-75.

6. W. Laskey, J. Boyle, L.W. Johnson, and the Registry Committee of the Society for Cardiac Angiography \& Intervention. Multivariable model for prediction of risk of significant complication during diagnostic cardiac catheterization. Cathet Cardiovasc Diagn 1993;30:185-90.

7. B. Chandrasekar, S. Doucet, L. Bilodeau, et al. Complications of cardiac catheterization in the current era: A single-center experience. Catheter Cardiovasc Interv. 2001;52:289-95.

8. H.D. Papaconstantinou, A.J. Marshall, C.J. Burrell. Diagnostic cardiac catheterisation in a hospital without on-site cardiac surgery. Heart 1999;81:465-9.

9. D. de Bono. Complications of cardiac catheterisation: results from 34041 patients in the UK confidential enquiry into cardiac catheter complications (CECCC). Br Heart J 1993;70:297-300. 
Journal of College of Medical Sciences-Nepal, 2012, Vol-8, No-2,

10. K.G. Oldroyd, K.V. Phadke, R. Phillips, et al. Cardiac catheterisation by the Judkins technique as an outpatient procedure. BMJ 1989;298:875-6.

11. J.E. Goss, C.E. Chambers, F.A. Heupler. Systemic anaphylactoid reactions to iodinated contrast media during cardiac catheterization procedures: guidelines for prevention, diagnosis, and treatment. Laboratory Performance Standards Committee of the Society for
Cardiac Angiography and Interventions. Catheter Cardiovasc Diagn. 1995;34:99-104.

12. C.L. Tomasso. Contrast induced nephrotoxicity in patients undergoing cardiac catheterization. Cath Cardiovasc Diagn. 1994;31:316-21.

13. B.J. Barrett. Contrast nephrotoxicity. JAm Soc Nephrol 1994; 5:125-37. 